\title{
Retrospective claims analysis of best supportive care costs and survival in a US metastatic renal cell population
}

This article was published in the following Dove Press journal:

ClinicoEconomics and Outcomes Research

8 July 2013

Number of times this article has been viewed

\author{
Henry J Henk' \\ Connie Chen ${ }^{2}$ \\ Agnes Benedict ${ }^{3}$ \\ Jane Sullivan' \\ April Teitelbaum' \\ 'Optum, Eden Prairie, MN, USA; \\ ${ }^{2}$ Pfizer Inc, New York, NY, USA; \\ ${ }^{3}$ United BioSource Corporation, \\ London, UK
}

Correspondence: Connie Chen

Pfizer Inc, 235 E 42nd St,

New York, NY 10017 , USA

Tel +I 2I27332650

Email connie.chen@pfizer.com
Introduction: Survival and best supportive care (BSC) costs for patients with metastatic renal cell carcinoma ( $\mathrm{mRCC}$ ), after stopping therapy, are poorly characterized yet an important aspect of patient care. This study examined survival and costs associated with BSC after one or two lines of therapy (LOTs) for mRCC.

Methods: A retrospective cohort analysis used claims data from commercially insured or Medicare Advantage Prescription Drug (MAPD) plan enrollees of a large United States health plan with an index RCC diagnosis (ICD-9-CM 189.0) between January 1, 2007 and June 30, 2010; initiating any of the following therapies 30 days pre-index date through disenrollment from plan: sunitinib, temsirolimus, sorafenib, bevacizumab, everolimus, pazopanib, cytokines. LOT was identified using prescription fill and administration dates. Health care costs represent health plan- plus patient-paid amounts.

Results: The cohort ( $\mathrm{n}=274)$ was $73 \%$ male, with a mean age of 63.3 years (SD 11.1), with $80 \%$ commercially insured (20\% MAPD), and 68\% starting BSC following one LOT. Mean BSC duration was longer following one than two LOTs (223 [SD 260], 176 [SD 163] days). Median survival from the start of BSC was similar following one and two LOTs (126 and 118 days). Total BSC costs following one and two LOTs averaged US\$50,188 (SD \$96,984) and \$37,295 (SD \$51,102). Monthly costs for BSC following one and two LOTs $(\$ 10,151$ and \$10,566) were not substantially lower than costs while on treatment $(\$ 14,621$ and $\$ 16,957)$. Inpatient hospital costs represented 47\% and 49\% following one and two LOTs, with ambulatory costs of approximately $36 \%$ following each LOT. Conclusion: Our study found similar survival and monthly costs for BSC following either one or two LOTs, with almost half of the cost reflecting inpatient care. Compared to costs on treatment ( $\$ 14,621$ to $\$ 16,957)$, BSC costs can be considerable $(\$ 10,151$ to $\$ 10,566)$.

Keywords: RCC, metastatic, supportive care, cost

\section{Introduction}

The American Cancer Society estimated that 65,150 individuals in the United States were expected to be diagnosed with renal cell carcinoma (RCC) in 2013, and that 13,680 would die from the disease. ${ }^{1}$ Renal cancer accounts for approximately $2-3 \%$ of all malignancies. More than $90 \%$ of kidney cancers are RCC (also called renal cell adenocarcinoma), and $85 \%$ of RCC are of clear-cell histology. ${ }^{2}$ The median age of RCC diagnosis is 65 years, and the greatest risk factors include smoking, obesity, and hypertension. ${ }^{3}$ The 5 -year relative survival rate for early-stage localized disease is $90.4 \%$, whereas the 5 -year relative survival rate for patients with later-stage disease involving distant metastases is substantially lower at $10.4 \%$. Since early disease is relatively asymptomatic, approximately $25 \%$ of RCCs are diagnosed in late stages. ${ }^{4}$ The most common metastasis sites include lung, bone, brain, and liver. ${ }^{2}$ 
Diagnosis of small and incidental renal cell cancer has become more common due to the frequent use of abdominal imaging modalities (eg, computed tomography, ultrasonography, and magnetic resonance imaging for evaluation of an unrelated problem). Many patients present with the classic triad of symptoms - flank pain, gross hematuria, and a palpable abdominal mass - that are further evaluated by imaging studies, and some patients present with manifestations of metastatic disease (eg, hypercalcemia due to bone metastases).

Surgery is the primary treatment recommendation for cancer stages I-III. ${ }^{2}$ For those with metastatic disease, cytoreductive nephrectomy may be part of a combined modality approach to decrease tumor bulk prior to administration of systemic therapy. Metastasectomy may be possible in patients with one or a limited number of metastases, and palliative nephrectomy may control severe local and systemic symptoms due to the primary tumor. ${ }^{5}$

The prognosis for patients with $\mathrm{mRCC}$ has improved in the last decade due to the introduction of targeted therapies that interfere with specific signal transduction pathways of tumor formation and progression, eg, blocking the vascular endothelial growth factor (VEGF) pathway, which is often overexpressed in RCC, and inhibiting tumor angiogenesis. ${ }^{4}$

The pathogenesis of clear-cell carcinoma is the best understood, and molecularly targeted therapy is the preferred treatment approach for most patients with advanced clearcell RCC. Therapies with anti-angiogenesic properties interrupt critical cell signaling pathways involved in tumor angiogenesis and growth. Prior to the development of molecularly targeted agents, immunotherapy with either interleukin (IL)-2 or interferon (IFN)- $\alpha$, or both, represented the primary treatment modality for patients with $\mathrm{mRCC}$, albeit at the cost of considerable toxicity without significant long-term clinical benefit. Sorafenib, approved in December 2005, and sunitinib, approved January 2006, were the first targeted therapies commercially available for treatment of mRCC. Sunitinib, sorafenib, axitinib, and pazopanib are US Food and Drug Administration (FDA)-approved tyrosine kinase inhibitors (TKIs) that also target VEGF receptors. ${ }^{6}$ Temsirolimus and everolimus are mammalian target of rapamycin (mTOR) kinase inhibitors. Bevacizumab is a monoclonal antibody that selectively binds the VEGF-A ligand; it is indicated for treatment of metastatic RCC in combination with IFN- $\alpha .{ }^{4}$ Several immunotherapy antibodies that target the family of immune checkpoint signaling molecules (PD-1) are currently in various stages of development and improved survival has been reported recently with patient-specific immunotherapy added to a TKI. ${ }^{7}$

Current guidelines from the National Comprehensive Cancer Network (NCCN), ${ }^{2}$ as well as the European Association of Urology (EAU) ${ }^{8}$ and European Society for Medical Oncology (ESMO) guidelines recommend sunitinib, axitinib, sorafenib, pazopanib, temsirolimus (in poor-prognosis patients in first-line therapy), everolimus, and bevacizumab + IFN as the main treatment options for the management of mRCC. NCCN also includes BSC in their list of recommended first-line therapies. NCCN uses the term "BSC" interchangeably with "palliative care," and describes palliative care as a organized method for controlling pain and other difficult symptoms that optimizes quality of life for the patient and family, irrespective of disease stage, and which may or may not be combined with life-prolonging therapies. ${ }^{9}$ Palliative care and BSC also encompass psychological and spiritual aspects of patient care, such as support systems to help the patient and family cope with the illness, side effects of treatment, and bereavement. ${ }^{10}$

The economic burden associated with treating RCC is substantial, but difficult to characterize, as evidenced by the wide range of cost estimates found across studies. ${ }^{11}$ With the development of targeted therapies for mRCC and an increasing number of treatment choices, it is important for decision makers to understand the costs associated with both active treatment and BSC, both during and following completion of treatment.

US studies typically estimate cancer costs by linking data from the Surveillance, Epidemiology, and End Results $(\mathrm{SEER})^{3}$ cancer registry to medical claims data. Yabroff et a ${ }^{12}$ estimated cancer costs for elderly patients during the period January 1, 1999 through December 31, 2003 (prior to targeted agents), based on linked SEER-Medicare data. They found mean total net costs (ie, difference in costs for patients with and without cancer) were approximately US $\$ 38,000$ in the last year of life for patients with RCC (all stages), and that over $70 \%$ of the costs were due to hospitalization. Furthermore, the mean total net costs in the last year of life were over $\$ 54,000$ for patients with an RCC diagnosis involving distant metastases. Yabroff et al examined aggregate cancer costs, so only reported on the cost of hospitalization because it was the primary driver of total cancer care costs. Other health care cost components were not reported. ${ }^{12}$

When examining cancer costs that are more specific to RCC, Lang et $\mathrm{al}^{13}$ estimated the burden of illness associated with RCC in the US in 2005 based on a prevalence model that used linked SEER-Medicare data from 1999 inflated to 
2005 dollars. They determined that the overall annual cost of RCC was US\$40,176 per patient, irrespective of age and disease stage. For patients who were $\geq 65$ years of age with distant metastases, the annual burden of RCC was \$28,271 per patient, and $\$ 26,573$ for health care costs alone. It is important to note that Lang et al used 1999 Medicare data from the pre-targeted therapy era, ${ }^{13}$ costs of newer targeted therapies would be higher. Also, although Lang et al reported costs for "additional resource utilization"13 (eg, nursing facility, hospice care), BSC care costs were not specifically addressed in the paper. A more recent study, examining the cost of treating RCC between 1995 and 2002, ${ }^{14}$ when IL-2 and IFN- $\alpha$ were standard practice, provides additional insights into BSC costs although not in the era of targeted therapies.

With an increasing number and variety of treatments available for mRCC, understanding the individual cost components involved in both treatment and BSC is important because of the implications for future health economic analyses. Furthermore, there appears to be a lack of published studies examining BSC costs for $\mathrm{mRCC}$ patients that specifically address the BSC components of health care costs. The objective of this study was to examine survival and costs associated with BSC after either one or two lines of mRCC treatment.

\section{Methods}

\section{Data sources}

\section{Claims data}

This was a retrospective claims database study using medical and pharmacy data and enrollment information from the Optum Research Database. The data included medical claims, pharmacy claims, and eligibility information from a large national US health plan that offers both commercial and Medicare Advantage Prescription Drugs (MAPD) insurance to approximately 14 million commercial and 500,000 Medicare Advantage enrollees per year. Enrollees have medical and pharmacy benefits and are geographically diverse across the US, with the greatest representation in the South and Midwest US census regions. Medical (professional, facility) claims included International Classification of Diseases, 9th Revision, Clinical Modification (ICD-9-CM) diagnosis codes, ICD-9 procedure codes, Current Procedural Terminology, Version 4 (CPT-4) procedure codes, Healthcare Common Procedure Coding System (HCPCS) procedure codes, and site of service codes. Outpatient pharmacy claims provided National Drug Codes for dispensed medications, quantity dispensed, drug strength, and number of days of supply.

\section{Mortality data}

Patient mortality and date of death were determined by linking the Social Security Administration (SSA) death master file ${ }^{15}$ to patient enrollment data. All study data were accessed using techniques compliant with the Health Insurance Portability and Accountability Act of 1996, ${ }^{16}$ and no identifiable protected health information was extracted during the course of the study. Because this study involved analysis of pre-existing, de-identified data, it was exempt from Institutional Review Board approval.

\section{Study subject identification}

Patients were considered for inclusion if they had a medical claim with an RCC diagnosis (ICD-9-CM diagnosis code 189.0) and evidence of metastatic disease between January 1 , 2007 and June 30, 2010. Metastatic disease was identified as initiation of any of the following treatments from 30 days prior to the first medical claim with an RCC diagnosis (defined as the index date) through disenrollment: sunitinib, sorafenib, pazopanib, temsirolimus, everolimus, bevacizumab, IFN- $\alpha$ $2 \mathrm{~b}$, or IL-2, as there is no approved targeted or chemotherapy treatment in early-stage RCC. Patients were required to be $\geq 20$ years of age, and have had two or more medical claims ( $\geq 7$ days apart) with an RCC diagnosis and 6 months of continuous enrollment prior to (pre) and following (post) the index date (patients disenrolling due to death $<6$ months post-index date were retained). Patients were excluded if they had more than one primary cancer from 30 days pre-index date through the index date +182 days. Finally, to avoid procedures performed as diagnosis "rule outs," claims from laboratories or diagnostic testing centers, or claims with CPT-4/HCPCS codes 36400-36425, 70010-76999, 78000-78799, 80008999 , S9529, or G0001 were not considered when identifying claims with cancer diagnosis codes.

\section{Lines of therapy (LOTs)}

An LOT refers to the treatment of RCC and was based on prescription fill and administration dates. The first LOT began with the initial date of the treatment administration or prescription fill, and ended with the start date of a second LOT minus 1 day. If there was no evidence for a second LOT and $>30$ days passed with no therapy, then the end date became the run-out date, the death date, or the disenrollment date. The same rule applied to subsequent LOTs. The initiation of a new therapy administration or prescription fill was evidence for a new LOT, except for bevacizumab in combination with IFN- $\alpha$ - or IL-2, while all other treatments were administered as monotherapy. 


\section{Study measures}

The patient demographics that were examined included: age, sex, insurance type, and geographic location in the US (Northeast, Midwest, South, and West).

\section{Outcomes}

The primary outcome measures in the follow-up period were BSC costs and survival following completion of one or two LOTs. We define BSC as not including chemotherapy, in line with NCCN palliative care guidelines. ${ }^{9}$ BSC began with the completion of a first LOT (BSC A) or second LOT (BSC B) where a new LOT did not follow, and ended with death or disenrollment. The costs considered during the BSC period represent health plan- plus patient-paid amounts and were further categorized as: inpatient (IP) costs (inclusive of all IP services); emergency room (ER); and retail pharmacy (Rx) costs (prescription medication including supportive care), ambulatory services (AMB) costs (administered supportive care drugs, diagnostics, and procedures provided in the physician's office or outpatient hospital setting excluding cost of IL-2 administered intravenously, but including cost of administering infusion), and other medical benefits costs (eg, skilled nursing facilities and home health). Costs of common treatments, eg, radiotherapy and pain relief applied during BSC are typically captured under AMB and Rx or may also be captured in the IP setting.

The secondary outcome measures were costs accrued during the period of time when patients were receiving a first or second LOT. The cost categories were identical to those described above for the BSC period. All costs are calculated and reported as mean total and per-patient-permonth (PPPM) costs. Costs were consumer price index (CPI) adjusted to 2010 .

\section{Statistical methods}

Descriptive statistics were calculated for baseline demographics and outcome measures. Means and standard deviations (SDs) were calculated for continuous measures, and $\mathrm{n}(\%)$ for categorical measures. Comparison of means was based on a $t$-test. Comparison of median survival by BSC cohort was based on the Kaplan-Meier estimator ${ }^{16}$ in order to account for varying lengths of follow-up due to disenrollment. Since health care costs often have SDs that equal or exceed the mean, due to the fact that health care costs usually follow a positively skewed distribution, we present both mean and median costs. All statistical analyses were performed using SAS version 9.1 (SAS Corporation,
Cary, NC, USA) and Stata version 10 SE (StataCorp, College Station, TX, USA).

\section{Results}

The results are reported separately for patients starting BSC following the first LOT (BSC A) or the second LOT (BSC B). Figure 1 displays the selection process for the sample of 670 RCC patients who met all inclusion criteria. Of the 670 RCC patients, 535 patients completed a first LOT while enrolled in the health plan, with 186 patients (34.8\%) transitioning to BSC and 18 patients dying. Of the remaining 349 patients who completed a first LOT and initiated a second LOT, 257 patients completed a second LOT while enrolled in the health plan, with 88 patients (34.2\%) transitioning to BSC and eight patients dying. The final study sample consisted of 274 patients, with 186 patients (67.9\%) who completed one LOT and transitioned to BSC (BSC A) and 88 patients (32.1\%) who completed two LOTs and transitioned to BSC (BSC B). The mean (median) duration of BSC was 223 (114) days following completion of one LOT and 176 (109) days following completion of two LOTs.

\section{Patient demographics}

Overall, the mean age was 63.3 years (SD: 11.1 years); $73 \%$ were male; $80 \%$ were commercially insured; and $78.5 \%$ resided in the Midwest or Southern regions of the US. Sunitinib was the targeted therapy in the prior LOT for $56 \%$ of the patients who transitioned to BSC A and $34 \%$ of the patients who transitioned to BSC B.

\section{Outcomes}

Figure 2 displays the median time on BSC (ie, length of survival from the start of BSC A [126 days, 95\% confidence interval: 75, 191] and from the start of BSC B [118 days, 95\% confidence interval: 92, 174]) when patients in the two groups accumulated BSC costs. Figure 3 displays the total health care costs incurred from the start of BSC A or $\mathrm{B}$, stratified by resource type (Figure 3A), and the PPPM total health care costs for each LOT and subsequent BSC (Figure 3B). Total health care costs during BSC A averaged $\$ 50,188$ (SD: $\$ 96,984$, median: $\$ 14,225$ ) and $\$ 37,294$ (SD: $\$ 51,101$, median: $\$ 19,822)$ during BSC B $(P=0.1512)$. IP costs accounted for $46.5 \%(\$ 23,328)$ of the total costs during BSC A and $48.7 \%(\$ 18,171)$ of the total costs during BSC B. Ambulatory costs accounted for $36.4 \%(\$ 18,258)$ of the total costs during BSC A and $36.9 \%(\$ 13,750)$ of the total costs during BSC B. In contrast, Rx, ER, and costs for other 


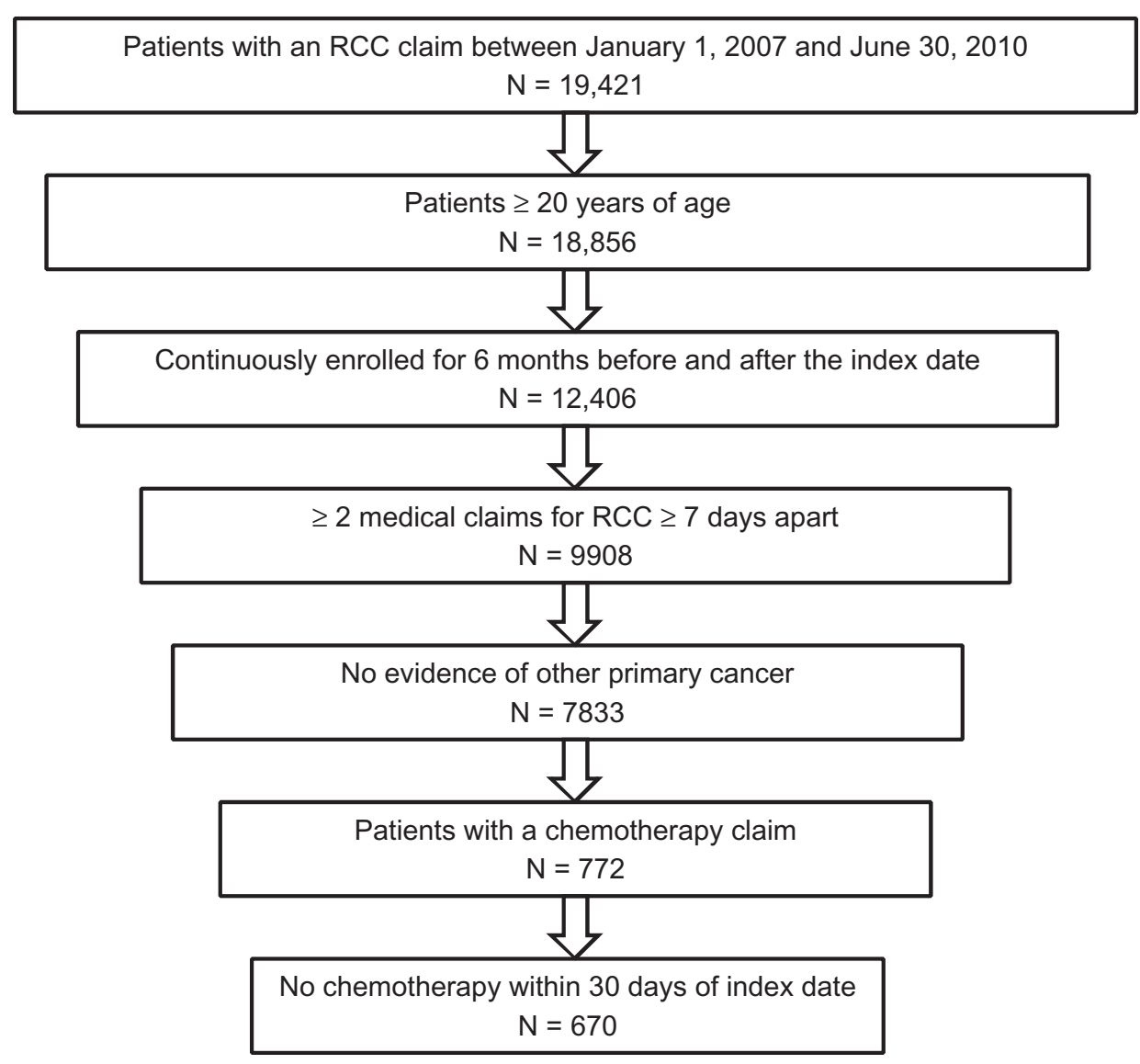

Figure I Sample selection process.

Abbreviation: RCC, renal cell carcinoma.

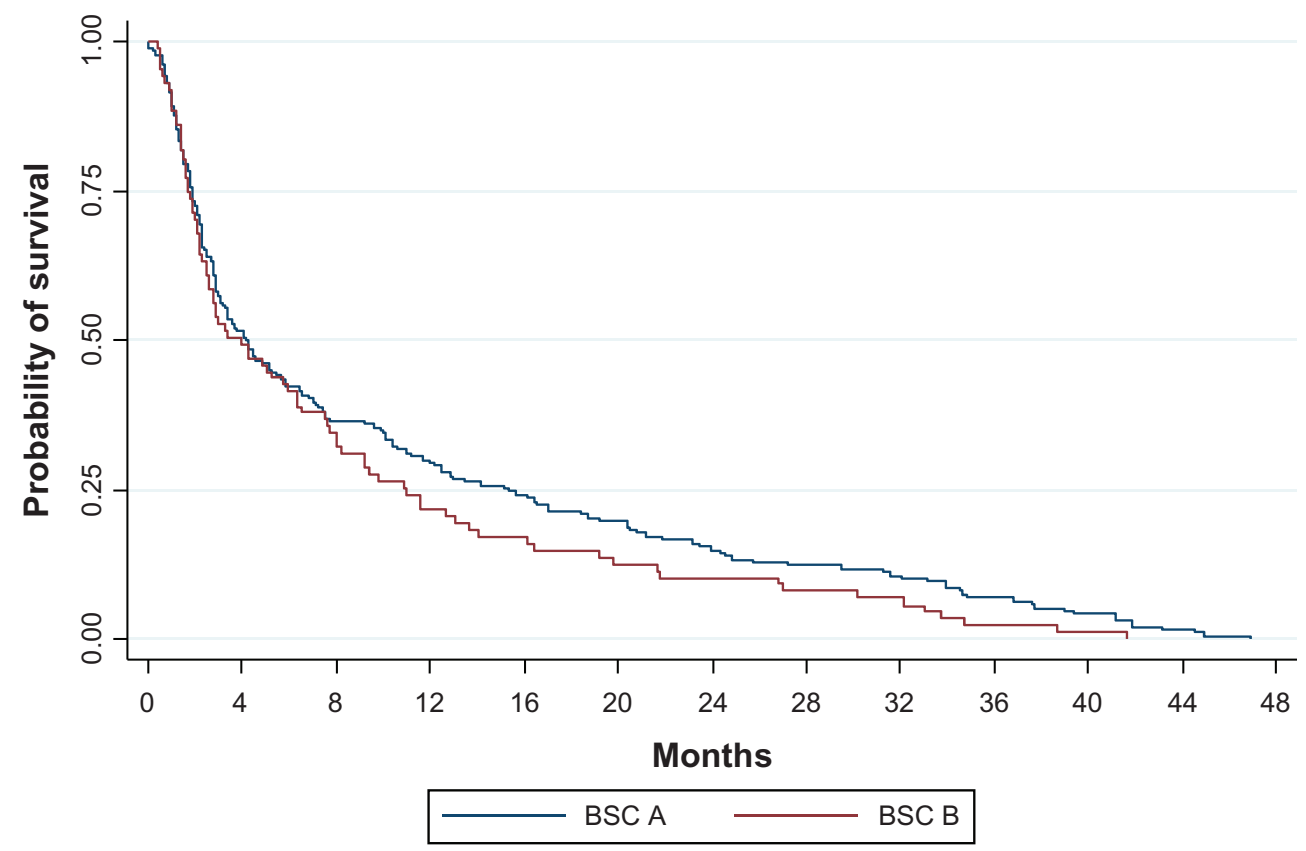

Figure 2 Kaplan-Meier survival estimates from start of BSC.

Notes: Median survival: 126 days and II 8 days for BSC A and BSC B, respectively $(P=0.1373)$.

Abbreviations: BSC, best supportive care; BSC A, BSC following first line of therapy; BSC B, BSC following second line of therapy. 
medical services accounted for a relatively small portion of the total costs during BSC A and BSC B. PPPM costs averaged \$14,621 (SD: \$18,501, median: \$8,306) during the first LOT and \$10,151 (SD: \$18178, median: \$2747) during BSC A following completion of one LOT. PPPM costs averaged \$16,957 (SD: \$45,416, median: \$8313) during the second LOT and \$10,566 (SD: \$17,648, median: \$4285) during BSC B following completion of two LOTs (Figure 3). While total costs during BSC differed following first and second LOTs, this is largely due to the somewhat longer duration of follow-up (ie, length of survival from start of BSC), thus PPPM costs are similar between BSC A and BSC B.

\section{Discussion}

This study examined the costs associated with BSC following the completion of one or two LOTs for mRCC. In the dataset considered, a significant proportion of patients (68\%) did not receive more than one LOT. Health care costs associated with BSC were proportionate to the duration of BSC. Also, in line with total costs reported in other studies, ${ }^{12,13,18}$ we found that the largest proportion of total BSC costs was associated with

A
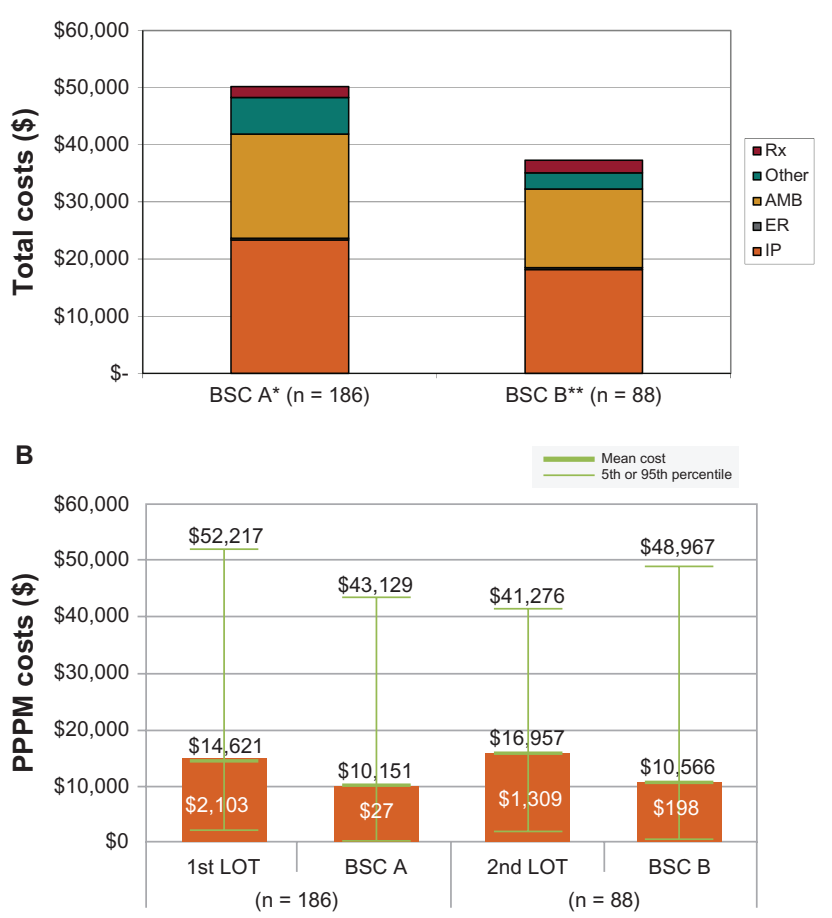

Figure 3 Total health care costs.

Notes: (A) From start of BSC; (B) PPPM. *Mean duration of BSC $=223$ days $($ median $=114)$. **Mean duration of BSC $=176$ days $($ median $=109)$. Costs were consumer price index adjusted to 2010 and are expressed in USD.

Abbreviations: AMB, ambulatory; BSC, best supportive care; BSC A, BSC following first line of therapy; BSC B, BSC following second line of therapy; ER, emergency room; IP, inpatient; LOT, line of therapy; PPPM, per patient per month; $\mathrm{Rx}$, prescription.
IP care following either one or two LOTs. We also examined median survival once starting BSC for the two groups, and found that survival from the start of BSC was similar for the two BSC cohorts. Note this does not imply similar overall survival from the start of the first LOT for the two groups. The secondary outcomes were the health care costs accrued during treatment versus subsequent BSC for patients receiving either one or two LOTs. Two findings emerge: (1) PPPM costs were higher during the second LOT than the first LOT; and (2) PPPM costs were $44.0 \%$ and $60.5 \%$ higher during LOT 1 and 2 (respectively) than during BSC following either LOT. IP costs were the greatest contributor to the PPPM BSC costs.

In our review of the literature, we found little information on $\mathrm{BSC}$ costs for patients with mRCC. Consequently, we believe that our study is one of the first comprehensive examinations of BSC costs following an active LOT in the era of targeted therapies in the US. These data are important for understanding the long-term economic consequences of a disease for which patients may be receiving BSC along with antitumor treatment for an extended duration. Data reported to date in economic analyses have been limited and variable, based mainly on physician interviews during the palliative care phase, despite the fact that BSC costs are often considered when determining cost-effectiveness of new targeted therapies (eg, Remak et $\mathrm{al}^{19}$ ). Of interest is a claims database analysis ${ }^{16}$ in the pre-targeted therapy era (1995 to 2002) where the estimated PPPM costs of treating $\mathrm{mRCC}$ patients in the first, second, and third year after diagnosis was about $\$ 7120, \$ 2600$, and $\$ 3060$, respectively (inflated to 2010 USD). If we assume that the progression-free interval was shorter in the pre-targeted therapy era, then the above estimates may largely reflect BSC services. If so, then BSC has become more resource intensive over time, as indicated by the current findings. However, this comparison is limited given differences in data sources and methods.

There are some limitations associated with retrospective studies that should be considered. The costs presented reflect amounts paid by the health plan and patient, and do not include amounts paid by other insurers, or societal costs in general. Furthermore, the results are based on a commercially insured population and may not be generalizable to other populations. However, it should be noted that $20 \%$ of the sample were Medicare Advantage enrollees and that approximately $24 \%$ of all commercially insured patients in this dataset were 65 years of age or older (primarily retirees) who most likely had some Medicare coverage. The combined $44 \%$ is in line with 2004 cost estimates from the National Cancer Institute 
showing that Medicare accounted for $45 \%$ of total treatment costs for all cancers. ${ }^{20}$ Also, it was assumed that treatment was for $\mathrm{mRCC}$ as no treatment is FDA-approved for an adjuvant setting. In actuality, there could be off-label or clinical trial use in the adjuvant setting. ${ }^{2}$ Since time on experimental agents would be captured as time on BSC, then BSC costs would be overestimated due to any overall survival benefit associated with the experimental treatments. Additionally, some or all of the active treatments received by patients enrolled in clinical trials may not generate insurance claims and therefore would not be included in the claims database. This study is also limited to the extent to which claims data can accurately capture an individual's medical history, and absence in the SSA file cannot be taken as proof that a person is alive. Furthermore, claims data are collected for the purposes of payment, and not for research. Presence of a diagnosis code on a medical claim is not an exact predictor of the presence of disease, as the diagnosis code may be incorrectly coded, or not recorded at all.

\section{Conclusion}

This study found similar survival and monthly costs for BSC following either one or two LOTs, with almost half of the cost reflecting IP care. Compared to costs on treatment $(\$ 14,621$ to $\$ 16,957)$, BSC costs can be considerable $(\$ 10,151$ to $\$ 10,566)$. This is the first study to report different BSC cost components and survival in patients with $\mathrm{mRCC}$ and therefore fills an important gap in our understanding of the economic burden of this disease in the US.

\section{Acknowledgment}

Virginia M Rosen, PhD is an employee of Optum and provided editorial/medical writing support.

\section{Author contribution}

All authors have made a significant contribution to the study design, data interpretation, and drafting of this manuscript which included critical revisions for important intellectual content. All authors have read and approved the final version for publication.

\section{Disclosure}

This study was sponsored by Pfizer Inc. Connie Chen is employed by and owns stock in Pfizer Inc. Optum received funding from Pfizer Inc in conjunction with the development of this study and manuscript. Henry J Henk, Jane Sullivan and April Teitelbaum are employees of Optum; Agnes Benedict is an employee of UnitedBioSource Corporation.

\section{References}

1. American Cancer Society: Cancer Facts and Figures 2013. Atlanta, GA: American Cancer Society; 2013. Available from: http://www.cancer.org/ research/cancerfactsstatistics/cancerfactsfigures2012/index. Accessed January 11, 2012.

2. NCCN Guidelines ${ }^{\circledR}$ : Kidney Cancer [webpage on the Internet]. National Comprehensive Cancer Network; 2012. Available from: http://www. nccn.org/professionals/physician_gls/f_guidelines.asp. Accessed November 3, 2011.

3. Howlader N, Noone AM, Krapcho M, et al, editors. SEER Cancer Statistics Review, 1975-2008 [webpage on the Internet]. Bethesda, MD: National Cancer Institute [updated 2011, based on November 2010 SEER data submission]. Available from: http://seer.cancer.gov/ csr/1975_2008/. Accessed November 3, 2011.

4. Kirchner H, Strumberg D, Bahl A, Overcamp F. Patient-based strategy systemic treatment of metastatic renal cell carcinoma. Expert Rev Anticancer Ther. 2010;10(4):585-596.

5. Escudier B, Albiges L, Sonpavde G. Optimal management of metastatic renal cell carcinoma: current status. Drugs. 2013;73(5):427-438.

6. Drugs@FDA [database on the Internet]. Silver Spring, MD: US Food and Drug Administration. Available from: http://www.accessdata.fda. gov/scripts/cder/drugsatfda/. Accessed January 11, 2012.

7. Amin A. Prolonged survival with personalized immunotherapy (AGS003 ) in combination with sunitinib in unfavorable risk metastatic RCC (mRCC). Abstract \#357. Presented at: Genitourinary Cancers Symposium; February 14-16, 2013; Orlando, FL.

8. Guidelines on Renal Cell Carcinoma: Systemic Therapy For Metastatic $R C C$. European Association of Urology; 2013. Available from: http://www. uroweb.org/guidelines/online-guidelines/. Accessed May 14, 2013.

9. NCCN Clinical Practice Guidelines in Oncology (NCCN Guidelines ${ }^{\mathrm{TM}}$ ). Palliative Care. Version 2.2011. National Comprehensive Cancer Network; 2011. Available from: http://www.oralcancerfoundation.org/ treatment/pdf/palliative.pdf. Accessed August 29, 2012.

10. Aapro MS. Supportive care and palliative care: a time for unity in diversity. Ann Oncol. 2012;23(8):1932-1934.

11. Shih YC, Chien CR, Xu Y, Pan IW, Smith GL, Buchholz TA. Economic burden of renal cell carcinoma: part I - an updated review. Pharmacoeconomics. 2011;29(4):315-329.

12. Yabroff KR, Lamont EB, Mariotto A, et al. Cost of care for elderly cancer patients in the United States. J Natl Cancer Inst. 2008;100(9): 630-641.

13. Lang K, Danchenko N, Gondek K, Schwartz B, Thompson D. The burden of illness associated with renal cell carcinoma in the United States. Urol Oncol. 2007;25(5):368-375.

14. Hollenbeak CS, Nikkel LE, Schaefer EW, Alemao E, Ghahramani N, Raman JD. Determinants of medicare all-cause costs among elderly patients with renal cell carcinoma. J Manag Care Pharm. 2011;17(8): 610-620.

15. Social Security Administration's Death Master File (DMF) [webpage on the Internet]. US Department of Commerce. Available from: http:// www.ntis.gov/products/ssa-dmf.aspx. Accessed September 1, 2011.

16. Health Insurance Portability and Accountability Act of 1996. Public Law 104-191. 104th Congress. 2009. Available from: http://www.cms.hhs.gov/ HIPAAGenInfo/Downloads/HIPAALaw.pdf. Accessed October 24, 2011.

17. Kaplan EL, Meier P. Nonparametric estimation from incomplete observations. J Am Stat Assoc. 1958;53:457-481.

18. Purmonen T, Nuttunen P, Vuorinen R, Pyrhönen S, Kataja V, Kellokumpu-Lehtinen P. Current and predicted cost of metastatic renal cell carcinoma in Finland. Acta Oncologica. 2010;49:837-843.

19. Remák E, Charbonneau C, Négrier S, Kim ST, Motzer RJ. Economic evaluation of sunitinib malate for the first-line treatment of metastatic renal cell carcinoma. J Clin Oncol. 2008;26(24):3995-4000.

20. Cancer and Medicare: A Chartbook. American Cancer Society; Cancer Action Network; 2009. Available from: http://www.allhealth.org/ briefingmaterials/CancerandMedicareChartbookFinalfulldocument March11-1412.pdf. Accessed September 4, 2012. 


\section{Publish your work in this journal}

ClinicoEconomics \& Outcomes Research is an international, peerreviewed open-access journal focusing on Health Technology Assessment, Pharmacoeconomics and Outcomes Research in the areas of diagnosis, medical devices, and clinical, surgical and pharmacological intervention. The economic impact of health policy and health systems

organization also constitute important areas of coverage. The manuscript management system is completely online and includes a very quick and fair peer-review system, which is all easy to use. Visit http://www.dovepress.com/testimonials.php to read real quotes from published authors.

Submit your manuscript here: http://www.dovepress.com/clinicoeconomics-and-outcomes-research-journal 